\title{
La conexión con el paciente en el siglo XXI
}

\author{
The connection with the patient in the 21 st century
}

\author{
María Elena Rivera Salazar ${ }^{1}$ \\ Rivera S. María E. \\ miradas $\mathrm{N}^{\circ} 14$ - 2016. ISSN: 0122 994X Págs 9 - 16 \\ Recepción: Junio 15 de 2016 \\ Aprobación: Octubre 03 de 2016 \\ Publicación: Diciembre 20 de 2016
}

\section{Resumen}

La conexión con el paciente, surge como una reflexión al cuestionamiento permanente como coordinadora de Medicina Interna en el programa de Medicina y como Directora de la Especialización en Medicina Interna durante siete años hasta el 2015. Preguntas, entre otras, como ¿qué debe aprender un estudiante en su proceso de formación como médico y para qué? Y la que más me inquieta: ¿estamos formando técnicos en medicina? El acto médico y la educación médica se han reducido a la aplicación de una información científica por parte de profesionales que se acuestan médicos y se levantan docentes. Es necesario reivindicar el hecho de comprender al paciente por parte del médico, del docente y del estudiante, como elemento vital en la formación del profesional médico; esto implica utilizar la tecnología como medio y no como fin y reconocer que detrás de toda enfermedad hay un rostro.

Palabras Clave: Educación médica, razonamiento clínico.

\begin{abstract}
The connection with the patient, emerges as a reflection to the permanent questioning during my role as Internal Medicine Coordinator at Medicine program and Internal Medicine Residency program for seven years until 2015. Questions, such as $¿$ what should learn a Student during training process as a doctor and what for? And the one that most worries me: are we training medical technicians? The medical act and medical education

1 Médico y Cirujano. Especialista en Pedagogía y Desarrollo Humano. Magister en Educación y Desarrollo Humano. Estudiante Doctorado en Ciencias de la Educación. Docente Medicina, Facultad Ciencias de la Salud, Universidad Tecnológica de Pereira.
\end{abstract}


have been reduced to the application of scientific information by professionals who go to bed being doctors and wake up as teachers. It is necessary to claim for patient empathy coming not only from the physician but the medical teacher and medical student, as a vital element in the training of the medical professional; This approach involves the use of technology as a tool and not as the end and to realize that behind every disease there is a face.

Key Words: Medical Education, clinical reasoning.

¿Para qué un programa de Medicina? ¿para qué estudiar medicina? ¿para qué formar médicos? ¿para qué hacer un plan de estudios? ¿por qué formamos médicos? ¿es la medicina una disciplina para promover la salud, prevenir y curar la enfermedad, rehabilitar al paciente y aliviar su sufrimiento? ¿qué debe aprender un estudiante en su proceso de formación como médico y para qué? ¿qué saberes además de los actuales en nuestro programa de medicina se requieren para lograr la formación integral del médico del siglo XXI? ¿cuál es la pertinencia de lo que enseño actualmente para la formación del futuro profesional de la medicina?

Responder esto es tarea compleja, por tanto no pretendo generar una lista de ingredientes para entregar una receta ideal.

Me surge una nueva pregunta: ¿estamos formando técnicos en medicina? A pesar de creer que los criterios que utilizamos para escoger nuestros nuevos admitidos, para mí no son los ideales, ingresan algunos estudiantes que a pesar de la pasión y amor que demuestran sentir para formarse como tal, no lo logran o se decepcionan ante el modelo que utiliza el docente para generar conocimiento con el alumno o para desarrollar juicio y razonamiento clínico o definitivamente para formarlo como médico.

Se genera entonces un contrato pedagógico virtual ante el premio anhelado "un título"; el estudiante finge que estudia y el docente simula que enseña.

Lo que veo es que el profesional "se acuesta médico y se levanta docente", por muchas razones que no voy a esbozar aquí; y es allí donde a pesar de tener conocimientos para promover la salud, prevenir la enfermedad, curar y rehabilitar, no dispone de las herramientas necesarias para moldear ese probable diamante que tiene al frente y utiliza la metodología de "la copia", tal cual le enseñaron sus profesores, tal cual quiere "transmitir el conocimiento" y termina así como un sinnúmero de "profesores", solo informando, coartando la posibilidad de pensar del estudiante, examinándolo con un instrumento que solo da cuenta de la memoria del educando, más no de su capacidad argumentativa, de análisis y menos aún de actuar en contexto.

La enseñanza de la medicina abarca muchos horizontes y se puede problematizar desde diferentes conceptos y metodologías. El Médico cumple un papel protagónico en la reflexión de la formación de profesionales en este campo. Mucho se ha especulado sobre la indiferencia de este profesional con la realidad social en la que vivimos.

Cuando empecé a analizar de manera crítica este aspecto, creí obligatorio pensar en el modelo de formación que han implementado las escuelas de medicina. Revisé la misión del programa de medicina de la Facultad Ciencias de la Salud de la Universidad Tecnológica de Pereira, el cual pretende "formar profesionales mediante el desarrollo de la docencia, la investigación y la proyección social, con docentes de alta calificación, inspirados en el servicio humanizado con el fin de contribuir a 
mejorar la calidad de vida de los seres humanos". (salud.utp.edu.co/medicina/ misión.html). Realicé recientemente una investigación con el fin de caracterizar los modelos pedagógicos que utilizan los docentes de Ciencias clínicas del programa de medicina de la mencionada institución y con esto quise aportar una primera aproximación a la realidad de la Educación en salud en ciencias clínicas de dicho programa, refiriéndome a modelo pedagógico como Pinilla (2011: 205) afirma:

Es la representación de las relaciones que predominan en los actos de enseñar, de aprender y de evaluar, en las características de la relación maestro- alumno, en la concepción que tenga el profesor de aprendizaje, de evaluación y en consecuencia cómo evalúa.

Hice referencia a cuatro modelos pedagógicos, como los más sobresalientes en Colombia y que delinean los planes curriculares de los programas de medicina: el modelo tradicional, el tecnológico, el social y un modelo reciente: el problematizador ${ }^{2}$. Los programas médicos en Colombia propiamente no siguen un modelo puro; se puede decir que se utilizan todos, aunque se tenga tendencia por uno más que por otro ( $c f$. Bohórquez, 2004: web). El resultado de mi estudio arrojó que la mayoría de los docentes de Ciencias clínicas, aunque combinan varios modelos, privilegian el modelo tecnológico para la enseñanza de su asignatura. Que esto sea lo pertinente para esta época, tendrá que ser motivo de otro análisis y documento.

2 Entendiéndose este último como el aprendizaje basado en problemas.
El médico se convierte en docente no necesariamente por su formación en educación o un talento innato, sino porque manifiesta un poder que le confiere el conocimiento adquirido por su estudio previo o por la práctica profesional. En el momento de evaluar esta transmisión de conocimiento con el alumno, lo único tangible es una prueba escrita u oral que es valorada de acuerdo al grado de similitud de términos que el estudiante manifieste en sus respuestas con lo que el profesor transfirió. Al presentar ese estudiante graduado a la sociedad, no es claro si las competencias adquiridas en la Universidad son suficientes para enfrentar la realidad.

Es posible que los médicos docentes utilicen en forma importante un método de enseñanza donde predomine la información sobre la verdadera formación y en algunas ocasiones faciliten y en otras restringen la formación integral del alumno, es decir, escatimen el moldeamiento global del alumno en sus dimensiones intelectuales, físicas, emocionales, sociales, espirituales.

La fuerte influencia de la enseñanza tradicional en el área de las ciencias naturales propicia encontrar teorías, tanto en los libros de texto como en las intervenciones orales de los profesores, como estructuras acabadas e inmutables. Es frecuente hallar, dentro de este enfoque tradicional, la presentación de leyes y fenómenos siguiendo rigurosos criterios lógico-deductivos. Estos dos aspectos hacen sólo referencia a la estructura de la ciencia enseñada, si a ellos integramos los provenientes de la intervención didáctica característica de los enfoques tradicionales de la enseñanza, queda claro 
que históricamente no se ha favorecido ni el conocimiento, ni la construcción, consciente y deliberada, de modelos mentales por parte de los alumnos. (Tamayo, 2013: 3486)

Al evaluar la evolución del estudiante semestre tras semestre, se respira a menudo en salas de profesores la necesidad de fortalecer competencias de los estudiantes en la elaboración pertinente de la historia clínica del paciente, en la realización del enfoque diagnóstico y en la adquisición de un razonamiento clínico adecuado. Apartándome de las capacidades con las que ingresa el estudiante al programa, recuerdo al Maestro en educación: Abraham Flexner ${ }^{3}$, quien afirmaba (Vicedo, 2002: 159), que "desde el punto de vista pedagógico, la medicina moderna, como toda enseñanza científica, se caracteriza por la actividad. El estudiante no solamente mira, oye y memoriza: hace. Su propio desempeño en el laboratorio y en la clínica son los principales factores de su instrucción y formación disciplinaria".

La formación del médico tiene además de la adquisición de bases sólidas teóricas, la aplicación de las mismas en un laboratorio, en una comunidad o en la práctica clínica ${ }^{4}$, apoyado en las herramientas que la tecnología le ofrece día a día. La atención médica y la formación del médico se deben centrar, por lo tanto en la conexión con el paciente en la práctica clínica, toda vez que es la pieza fundamental de la relación del médico con su paciente; de otro modo, es posible que el no estar dirigiendo todo el esfuerzo pedagógico para utilizar esta herramienta, alimente la deshumanización de la medicina y la indiferencia del médico 3 Nacido en Louisville, Kentucky, el 13 de noviembre de 1866, graduado de artes y humanidades de Johns Hopkins University y con especialización en educación de la escuela de graduados de Harvard

4 Entendida esta práctica como la comunicación del médico con su paciente en su lecho de enfermo. con su entorno social. Tal como lo dijo Sir William Osler $^{5}$ en su libro The principles and Practice of Medicine: "Estudiar la medicina sin libros, es como navegar un mar sin carta; y estudiarla solamente en los libros, equivale a no saber navegar".

De manera que el contacto con la persona enferma o que busca atención médica, es vital para la formación del estudiante. Adquirir habilidades comunicativas que le permitan interactuar con el paciente para obtener la información necesaria sobre su sintomatología o signología es fundamental en la formación de un excelente profesional. No concibo un buen diagnóstico, sin una buena semiología médica. Aproximarse al mismo, dependerá entonces de la capacidad comunicativa adquirida por el estudiante, del desarrollo de un juicio y razonamiento clínicos pertinentes y de conocer en qué contexto sociocultural vive el paciente.

Existe un divorcio entre la Universidad y la sociedad. Este es un diagnóstico intuido, conversado, reclamado por empresas, centros de desarrollo tecnológico, organizaciones de sociedad Civil, egresados y aún estudiantes activos. ¿Cuál es el verdadero norte de las instituciones dedicadas a formar profesionales? es prioritario hacer una reingeniería al conocimiento adquirido dentro del campus, tomando en cuenta los entes externos, formando redes y alianzas estratégicas, proyectándonos a la sociedad, en conclusión, culturizando la Universidad.

Entre tanto, ¿cómo puedo lograr esto? ¿cómo puedo atenuar el modelo tradicional en el que predominan las explicaciones de los maestros, las exposiciones monótonas, la magistralidad, la autoridad, el silencio y la modorra? ¿cómo puedo lograr un escenario donde la participación activa y alegre de los estudiantes permita inferir,

5 Sir William Osler, médico canadiense, padre de la medicina moderna en su libro the principles and Practice of Medicina. 
analizar, deducir, discutir, argumentar, crear, inventar, innovar, descubrir, en fin, que le permita pensar en este tiempo líquido? ${ }^{6}$

Hoy está disponible un nuevo modelo pedagógico por competencias con enfoque constructivista que rompe con la lógica de la trasmisión pura y simple de conocimientos, a favor de la adquisición de un nuevo saber a partir de la práctica en un contexto dado. Ahora se requiere reducir el número de contenidos y que bueno sería no hablar de contenidos, para no pensar en contenedores y así dar tiempo a la reflexión y a la indagación en el trabajo mediante enigmas, debates, situaciones-problemas, pequeños proyectos de investigación, observación, experimentación, etc. El plan de estudios debe ser un guión ${ }^{7}$ donde tanto el profesor como el alumno o alumna sean actores que sepan salir airosos de la complejidad del día a día, improvisando exitosamente en buena parte de los casos. Parodiando a Poper $^{8}$ quien dijo que las leyes de un país deben ser tan bien hechas, que una persona malosa no haga mucho daño, debemos hacer un currículo que evite al profesor deformar, en favor de formar $y$ transformar al estudiante y en el cual, lo oculto no afecte al estudiante.

Por consiguiente, todo estudiante requiere aprender a aprender, a desaprender, a buscar y seleccionar la información, a ser un lector con pensamiento crítico que cuestione la literatura y los paradigmas científicos. Lo anterior, le permitirá al nuevo profesional generar soluciones para diferentes problemas de diversa complejidad. (Pinilla, 2011: 276).

6 En palabras de Zygmunt Bauman

7 En palabras del Doctor Julián Serna: Filósofo.

8 Filósofo austriaco
Se hace necesario entonces dar al estudiante mas oportunidades durante su práctica con el docente, para discutir sus experiencias y preocupaciones e integrar sus vivencias clínicas con el aprendizaje basado en competencias bajo la tutoría de un profesor y con tiempo suficiente para que lo haga de manera adecuada y segura; siempre y cuando ese estudiante se encuentre guiado constantemente por el docente quien en el camino, será el instrumento facilitador de la transformación del alumno. ¿Cómo formar a este docente para que desarrolle su labor como debe ser? ¿Cuál es el deber ser del docente?

Yo sueño con un docente que valore al estudiante y al paciente como persona, que no centre su enseñanza en la enfermedad sino en el sujeto que busca su consuelo y en el estudiante que anhela aprendizaje; que reconozca el contexto sociopolítico en el que se desenvuelve; que valore la interdisciplinariedad y le asigne la importancia necesaria a la investigación para poder seguir desarrollando la ciencia médica. Esto probablemente redignificará una profesión que vuelve a vivir la esclavitud de otrora y de ahora, al servicio de la politiquería de turno. ¿Cómo he de transformar un médico en educador? ¿pueden todos los médicos ser educadores? ¿Cómo educar desde un equilibrio entre profesor y alumno, para beneficio del paciente y la sociedad? ¿cómo educar desde las emociones y las pasiones con las que llega el estudiante a la Universidad?

Estoy de acuerdo en que la Educación en Colombia y la educación médica, se encuentran atravesando una gran crisis y que los aspirantes a médicos estén aprendiendo lo que deben, fuera del cubículo o escenario universitario y en muchas ocasiones, lejos del profesor. Muñoz (2015: 5) afirma:

Como consecuencia de las más recientes tendencias innovadoras a nivel mundial, la conjunción de componentes tecnológicos de 
punta en los sectores de la tele-información, la informática y la comunicación de masas trajo consigo esa especie de revolución que hoy impregna el tránsito al nuevo milenio y de la que hacen parte fenómenos como el de las culturas electrónicas mass-mediáticas, los espacios virtuales que amplían sin fronteras la cosmovisión del entorno socio cultural, los nuevos escenarios de prácticas simbólicas y reproductivas que obligan a replantear las tradicionales funciones de instituciones como la familia y la escuela. Ahí media la comunicación como elemento clave de esa recomposición estructural de la vida colectiva, y en particular, sobresale la importancia que reviste la relación Comunicación-Educación como fuente de nuevos derroteros capaces de contribuir positivamente a construir un proyecto de país.

Pienso y repito constantemente a mis alumnos : "detrás de toda enfermedad hay un rostro", "detrás de toda enfermedad hay una comunidad", "detrás de toda enfermedad, hay una cultura". ¿Cómo considerar al estudiante un actor dinámico? o mejor: ¿Cómo volver al profesor un protagonista activo? El darle mayor peso a la interactividad y conectividad no están logrando esto en la educación médica. Si bien ha facilitado el camino, su abuso e idolatrización ha hipertrofiado un modelo tecnológico que refuerza mi impresión diagnóstica de estar formando "técnicos en medicina".

Continúan los cuestionamientos en mi mente: ¿se podrá pensar en algún momento en la homeostasis del asunto? ¿puede en algún momento la administración estar al servicio de la academia? ¿cómo hace el multiplicador para no dañar la emoción ni el magnetismo del otro cuando él mismo ya viene con el suyo averiado? ¿cómo formatear al presunto profesor que se acuesta médico y amanece docente? ¿cómo educar en serio y no en serie? ¿cómo alimentar las pasiones con las que llega el estudiante al aula con profesores de mentalidad plana? ¿por qué el pensamiento cartesiano sigue dominando y continuamos rodeados de "médicos" morbo-céntricos? ¿se requiere crear una asignatura que se llame Comunicación en salud para visibilizar la necesidad de mejorar en este aspecto? ¿se requiere crear una asignatura o módulo que se nombre Razonamiento clínico, para evidenciar la necesidad de aprendizaje del alumno y porque no decirlo del profesor en este campo? El acto médico y el acto Educativo-médico no pueden seguir siendo reducidos a la aplicación de una información científica; se requiere este proceso de razonamiento.

Enseñar razonamiento clínico agudizará el criterio "ojo" clínico del futuro médico al presentársele ejemplos ilustrativos y analizarlos de manera crítica. El razonamiento clínico resume el proceso de análisis que realiza el médico de los datos obtenidos del paciente tanto en el interrogatorio minucioso como en el hallazgo de signos importantes al examen físico, complementando con ayudas diagnósticas que podrán confirmar, descartar o reorientar una presunción diagnóstica para finalmente orientar una conducta terapéutica que finalmente alivie el dolor, de calidad de vida al enfermo y justamente no haga más daño del que el paciente tiene.

El razonamiento clínico no se aprende observando como otros lo hacen; este se aprende en problemas o casos clínicos cuidadosamente seleccionados para discutir con médicos y estudiantes, de tal modo que estos últimos tengan la oportunidad de realizar el proceso de discusión y argumentación previo análisis de la historia clínica del paciente (síntomas y signos), para concluir con un diagnóstico presuntivo y una decisión terapéutica favorable para el paciente. "Hierro con 
hierro se aguza, hombre con hombre se afila"9

Dado que las teorías existentes sobre la cognición sólo son suficientes para proporcionar una descripción provisional del razonamiento clínico, suplementamos la descripción de los principios con el método que utilizamos para aprender los fenómenos médicos, es decir, el aprendizaje por la creación de ejemplares o copias (o sea, mediante ejemplos). (Kassirer, Wong y Kopelman,2010, p. vii).

De esta manera minimizamos los errores médicos al mejorar la capacidad de interpretación del paciente y sus quejas o dolencias.

En algunos casos, la
generación de hipótesis
defectuosas puede atribuirse a
una interpretación inapropiada
de los signos clínicos, a fallos
en la identificación adecuada
de los signos para sospechar
una determinada enfermedad,
o a falta de conocimientos
para evocar una enfermedad.
(Kassirer et al., 2010: 39 )

Son muchos los errores médicos actuales entre los más comunes están: deficiente comunicación del médico con su paciente, deficiente comunicación entre el personal médico a cargo del paciente, personal insuficiente, fallo del material, inadecuada interpretación de las órdenes médicas, uso equivocado de fármacos y pruebas de laboratorio, error en la interpretación de las ayudas diagnósticas, diagnósticos erróneos. Sumado a esto la austeridad educativa actual: muchos pupilos en un aula recibiendo spray de mucho, para rociar

9 Proverbios 27:17 las neuronas de todo y así terminar como prescriptores de recomendaciones de moda. Entre este desangre de la profesión médica y el intento por solidificarla, entre disuelve y coagula, viene entonces el importante papel de la hemostasia educativa; a este nuevo saber hay que ponerle piel y corazón; hay que ponerle PACIENTE. Yo vivo en una comunidad que bien pudiera estar sufriendo el sindrome de Hubris; medio dioses imponiendo verdades absolutas y gritando sus dogmas por doquier; no vale el argumento, no vale la emoción, se ignora el ser político, se obnubila la sociedad; se anula el SER.

Revisar nuevamente algunas teorías del aprendizaje; refinar, reafirmar y afinar otras, me permite darle más fuerza a mi mente para persistir en mi intento de revivir la clínica, entendida esta como el aprendizaje y enseñanza del diagnóstico al lado del paciente y con el paciente a través del relato que este hace de su sintomatología y de los signos detectados por el médico al realizar el examen físico del paciente. Pensar esto hoy en un medio tecnofílico, bien puede ser una herejía educativa y profesional; de tal manera me puedo declarar tranquilamente, heresiarca.

Se necesitan el resurgimiento de verdaderos profesores, que modelen $\mathrm{y}$ moldeen estudiantes motivados, pensantes. Médicos profesores que enseñen a pensar e interpretar los síntomas y signos del paciente; el médico piensa, la tecnología no. Hipócrates del siglo XXI, que reaviven la esencia de quien solía decir: "es más importante saber que tipo de persona tiene la enfermedad, que saber que tipo de enfermedad tiene la persona" $"$. Hoy más que nunca se requiere reanimar la práctica clínica. La tecnología nos ayuda a confirmar o descartar de manera aproximada sospechas diagnósticas, pero no plantea hipótesis. Por esta razón

10 Recuperado de htpp://www.aforismos.net/autor/ hipocrates 
la clínica jamás podrá ser reemplazada por la tecnología. Afortunadamente este pensamiento ha sido compartido por otros clínicos e investigadores, entre ellos Alvan R. Feinstein quien antes de fallecer en el año 2001, dejó esta importante sentencia: "el médico que teme ser sustituido por una computadora, merece ser sustituido por una computadora". Es por tanto el médico quien posee el arte de interpretar al paciente, lograr una impresión diagnóstica y propender al mejoramiento de su calidad de vida.

Siguen vigentes las palabras que escribió T.S.Eliot hace tres cuartos de siglo, en su poema "The Rock": "Where is the wisdom we have lost in knowledge? Where is the knowledge we have lost in information? “¿Dónde está la sabiduría que hemos perdido en los conocimientos? ¿Dónde están los conocimientos que hemos perdido en la información?

\section{Referencia Bibliográfica}

Argüello A., Sandoval C. (2006). El sistema de Educación Médica en Colombia: cien años de atraso. Recuperado de http://revistas. urosario.edu.co/index.php/ revsalud/article/viewFile/543/467

Bohórquez G. Francisco, Gutiérrez Elio Fabio. (2004). "Modelos pedagógicos y cambios curriculares en Medicina. Una mirada crítica". Revista Facultad de ciencias de la Salud universidad del Cauca, núm. 2, Recuperado de http:// www.facultadsalud.unicauca. edu.co/revista/backweb/Revista/ Revista/0602062004.html
Cano Echeverry Margarita María, Pinzón Duque Oscar Alonso, Rivera Salazar María Elena. (2005). "Comprensión de los criterios morales que subyacen la práctica médica de los médicos en formación del programa de Medicina de la Universidad Tecnológica de Pereira". Revista Médica de Risaralda, Vol. 11 núm. 2.

Kassirer, Jerome P. , Wong, John B., Kopelman, Richard I. (2011). Manual de razonamiento clínico. Barcelona,España: Ed. Wolters Kluwer heallth España S.A., Lippincott Williams \& Wilkins.

Muñoz González, Germán. (2015). Genealogía del campo EducaciónComunicación-Cultura en Colombia.

Pinilla Análida Elizabeth. (2011). "Modelos pedagógicos y formación de profesionales en el área de la salud". Acta médica colombiana. Vol. $36 \mathrm{~N}^{\mathrm{o}} 4$.

Pinzón Duque Oscar Alonso, Rivera Salazar María Elena. (2004). "Algunas reflexiones sobre el papel de la historia en la formación de los médicos del siglo XXI". Revista Médica de Risaralda.

Tamayo Alzate, Óscar Eugenio. (2013). "Modelos y modelización en la enseñanza y el aprendizaje de las ciencias". IX congreso internacional sobre investigación en didáctica de las ciencias.

Vicedo Tomey, Agustín. (2002). “Abraham Flexner, pionero de la educación médica". Rev Cubana Educ Med super, pp. 156-163. 